\title{
A deep wide survey of faint low surface brightness galaxies in the direction of the Coma cluster of galaxies ${ }^{\star}$
}

\author{
C. Adami ${ }^{1}$, R. Scheidegger ${ }^{2}$, M. Ulmer ${ }^{2}$, F. Durret ${ }^{3,4}$, A. Mazure ${ }^{1}$, M. J. West ${ }^{5}$, \\ C. J. Conselice ${ }^{6}$, M. Gregg ${ }^{7}$, S. Kasun ${ }^{2}$, R. Pelló ${ }^{8}$, and J. P. Picat ${ }^{8}$ \\ 2 Department of Physics and Astronomy, Northwestern University, 2131 Sheridan Road, Evanston IL 60208-2900, USA \\ 3 Institut d'Astrophysique de Paris, CNRS, Université Pierre et Marie Curie, 98bis Bd. Arago, 75014 Paris, France \\ 5 Department of Physics and Astronomy, University of Hawaii, 200 West Kawili Street, LS2, Hilo HI 96720-4091, USA \\ 7 Department of Physics, University of California at Davis, 1 Shields Avenue, Davis, CA 95616, USA
}

1 LAM, Traverse du Siphon, 13012 Marseille, France e-mail: christophe.adami@oamp. fr

4 Observatoire de Paris, LERMA, 61 Av. de l'Observatoire, 75014 Paris, France

${ }^{6}$ Department of Astronomy, Caltech, MS 105-24, Pasadena CA 91125, USA

${ }^{8}$ Observatoire Midi-Pyrénées, 14 Av. Edouard Belin, 31400 Toulouse, France

Accepted 4 July 2005 / Received 28 August 2006

\section{ABSTRACT}

\begin{abstract}
Aims. We report on a search for faint ( $R$ total magnitude fainter than 21$)$ and low surface brightness galaxies $(R$ central surface brightness fainter than $\sim 24$ ) (fLSBs) in a $0.72 \times 0.82 \mathrm{deg}^{2}$ area centered on the Coma cluster.

Methods. We analyzed deep $B$ and $R$ band CCD imaging obtained using the CFH12K camera at CFHT and found $735 \mathrm{fLSBs}$. The total $B$ magnitudes, at the Coma cluster redshift, range from -13 to -9 with $B$ central surface brightness as faint as $27 \mathrm{mag}^{-2} \operatorname{arcsec}{ }^{-2}$. Results. Using empty field comparisons, we show that most of these fLSBs are probably inside the Coma cluster. We present the results of comparing the projected fLSB distributions with the distributions of normal galaxies and with known X-ray over densities. We also investigate their projected distribution relative to their location in the color magnitude relation. Colors of fLSBs vary between $B-R \sim 0.8$ and $\sim 1.4$ for $2 / 3$ of the sample and this part is consistent with the known CMR red-sequence for bright $(R \leq 18)$ ellipticals in Coma.

Conclusions. These fLSBs are likely to have followed the same evolution as giant ellipticals, which is consistent with a simple feedback/collapse formation and a passive evolution. These fLSBs are mainly clustered around NGC 4889. We found two other distinct fLSB populations. These populations have respectively redder and bluer colors compared to the giant elliptical red-sequence and possibly formed from stripped faint ellipticals and material stripped from spiral in-falling galaxies.
\end{abstract}

Key words. galaxies: clusters: individual: Coma

\section{Introduction}

In the last three decades, surveys of the local universe have revealed the presence of galaxies only a few percent brighter than the sky background, known as Low Surface Brightness (LSB) galaxies. LSBs have remained mostly undetected because galaxy detection is contaminated by the brightness of the night sky. Little is known about LSBs: their origin, physical properties (e.g. luminosity, colors, radius) and number density remain enigmatic. Because of the fundamental difficulty in detecting LSBs, it is also possible that some types of LSBs are still unknown. A number of studies have been carried out to identify LSBs and study their origin but no clear scheme of formation and evolution has been favored (e.g. Binggeli et al. 1985; Schombert et al. 1992; Bothun et al. 1993; Bernstein et al. 1995; Impey et al. 1996; Sprayberry et al. 1996; Ulmer et al. 1996, hereafter U96; Impey \& Bothun 1997; O’Neil et al. 1997; Kuzio de Naray et al. 2004; Sabatini et al. 2005). The present paper is devoted

* Based on observations obtained at the Canada-France-Hawaii Telescope (CFHT) which is operated by the National Research Council of Canada, the Institut National des Sciences de l'Univers of the Centre National de la Recherche Scientifique of France, and the University of Hawaii. to the search for faint Low Surface Brightness galaxies (fLSBs) in a cluster environment and to a discussion of their origin and properties.

LSB galaxies are commonly defined by a central surface brightness fainter than 22 or 23 mag per square arcsec in the $B$ band (cf. Bothun et al. 1991). Throughout this paper, we are interested in fainter objects with the following definition: galaxies fainter than $R=21$ (absolute $R$ magnitude fainter than $\sim-14$ at the distance of the Coma cluster), with radius larger than $\sim 0.6$ arcsec (slightly less than $3 \mathrm{kpc}$ ) and with $R$ central surface brightness fainter than $\sim 24 \mathrm{mag} \operatorname{arcsec}^{-2}$. The radius corresponds here to the standard deviation of the Gaussian fit to the surface brightness profile of the fLSBs (see Sects. 2.3 and 2.4).

Further studies of fLSBs and their properties are needed for several reasons:

First, according to Cold Dark Matter (CDM) models of hierarchical structure formation (White \& Rees 1978; White \& Frenk 1991), there should be abundant low-mass dark matter halos present in the Universe. These halos could develop low luminosity stellar systems and be detected as low luminosity galaxies. However, CDM theory overestimates the number of such detected dark halos: observations have reported far fewer low luminosity galaxies than predicted by simulations (e.g. 
Davies et al. 2004; but also see Kravtsov et al. 2004, for possible alternative solutions). A simple explanation for this discrepancy is that these numerous low luminosity galaxies exist but are too faint to be detected. Because fLSBs are strongly dominated by Dark Matter (e.g. McGaugh et al. 2001; de Blok et al. 2001) and are by definition the most difficult low luminosity galaxies to detect, they are the perfect candidate to fill the apparent lack of low luminosity structures.

Second, another difference between CDM theory and observations is the so-called "dwarf to giant ratio" in different environments. According to CDM models, low luminosity galaxies should be present and similar in all environments. The rich galaxy clusters such as Coma, Fornax, and Virgo have a substantial low luminosity population illustrated by a high dwarf to giant ratio (e.g. Secker et al. 1997; Roberts et al. 2004; Sabatini et al. 2005), but a possible lack of faint/dwarf galaxies has been reported in lower density environments such as the Local Group (e.g. Mateo 1998). To reconcile observations with theory, low luminosity galaxies must be selectively destroyed or transformed in low density environments and/or maintained or created in clusters of galaxies. fLSBs being among the most sensitive galaxies to environment-dependent processes, their studies in different environments is crucial.

In this paper, we report on an extensive new study of fLSBs in the Coma cluster. Rich environments can be harsh to dwarf galaxies and LSBs (e.g. López-Cruz et al. 1997; Gregg \& West 1998). Cluster galaxies can be affected by various processes that are not so often at play for field galaxies: direct collisions, tidal interactions, high speed encounters, ram pressure stripping by the intracluster medium (ICM), pressure confinement and combinations of the above. Pressure confinement (e.g. Babul \& Rees 1992), however, does not work for galaxies moving through the ICM at the typical velocity of rich clusters $\left(\geq 800 \mathrm{~km} \mathrm{~s}^{-1}\right)$, where the effects of ram pressure become important.

The Coma cluster is one of the densest nearby rich clusters and is therefore excellent for studying the effects of environment on the formation and evolution of galaxies. It also has the advantage of being located near the North Galactic pole, which makes the effects of galactic absorption negligible.

Coma has been extensively studied in the literature (see Biviano 1998, for a review of works before 1995) and is a complicated cluster, with evidence for several mergers (see recent reviews in "Merging Processes in Galaxy Clusters" 2002, Feretti et al., ed. Kluwer). It also contains two D (or one $\mathrm{cD}$ and one D galaxies, see Schombert et al. 1992; López-Cruz et al. 1997), $\mathrm{X}$-ray emission with strong substructures (Neumann et al. 2003), an extended radio halo (Giovannini et al. 1993) and a radio relic (e.g. Feretti \& Neumann 2006). Despite the wealth of observations on the Coma cluster, most works at optical wavelengths were limited to relatively bright magnitudes (a few examples are Andreon \& Cuillandre 2002; Beijersbegren et al. 2002; Iglesias-Páramo et al. 2003; and Lobo et al. 1997) or to relatively small areas with limited spectral coverage (e.g. Trentham 1998, with only two bands and $0.19 \mathrm{deg}^{2}$; or Bernstein et al. 1995, with a single deep band and $0.0145 \mathrm{deg}^{2}$ ). Our data (Adami et al. 2006, hereafter A06) fill these gaps since they are at the same time wide $\left(0.72 \times 0.82 \mathrm{deg}^{2}\right.$ or $\left.1.8 \mathrm{Mpc}^{2}\right)$, deep $(R \sim 24)$ and with a large wavelength coverage $(B, V, R$, and $I$ bands with equivalent depths).

The paper is organized as follows: we present the observations and the fLSB detection algorithm in Sect. 2. In Sect. 3 we compare our galaxy sample with other surveys. In Sect. 4, we discuss the fLSB colors. In Sect. 5 we discuss the spatial distribution of the fLSBs and its relation with that of the giant galaxies. In Sect. 6 we discuss our results in terms of some possible mechanisms that occur specifically in the cluster and give our conclusions in Sect. 7.

We assume a distance to Coma of $95 \mathrm{Mpc}, H_{0}=$ $75 \mathrm{~km} \mathrm{~s}^{-1} \mathrm{Mpc}^{-1}, \Omega_{\Lambda}=0.7, \Omega_{\mathrm{m}}=0.3$, distance modulus $=$ 34.89 , and therefore the scale is $0.44 \mathrm{kpc} \operatorname{arcsec}^{-1}$. All magnitudes are given in the Vega system.

\section{Observations and data analysis}

\subsection{Observations}

The observations are described in A06 and we only reproduce the salient points in this section. The Coma field was observed in April 1999 and April 2000 with the Canada-France-Hawaii $3.6 \mathrm{~m}$ telescope using the $\mathrm{CFH} 12 \mathrm{~K}$ camera. This camera is a mosaic of twelve CCDs. Two sets of images were taken in order to cover both the north and south regions of the cluster. The entire observed field covers an area of $0.72 \times 0.82 \mathrm{deg}^{2}$ centered on the two giant elliptical galaxies NGC 4874 and NGC 4889 at the core of the Coma cluster. Images were acquired using $B, V$, $R$ and $I$ Johnson-like filters. The seeing ranged from $0.9 \operatorname{arcsec}$ in $R$ to 1.07 arcsec in $B$. One pixel corresponds to 0.206 arcsec.

Because the goal of this work was to detect fLSBs in the deepest band $(R)$ and then to investigate the distribution of fLSBs in a color magnitude relation, we only used the $R$ and $B$ bands. We will use other bands (including $U$ band data that we plan to acquire) to study spectrophotometric properties of fLSBs in a future work.

The data reduction specific to this project was a three step process: step 1 was to search for all the objects in the $R$ image using SExtractor (Bertin \& Arnouts 1996); step 2 was to select all the faint low surface brightness objects from the SExtractor catalog; and, step 3 was to derive the magnitudes for the selected fLSBs in both $R$ and $B$ bands and to derive colors from photometry in the same aperture.

\subsection{Step 1: detection of sources}

We used SExtractor to detect all objects on the $R$ images, classify them as stars or galaxies and calculate among other things their total magnitude, core magnitude and coordinates. Object detection with SExtractor was not optimized to detect low surface brightness objects. The resulting SExtractor catalog contains over 60000 detections, including stars, globular clusters, galaxies, etc.

\subsection{Step 2: identification of low surface brightness galaxies}

The second step of the analysis was to identify fLSBs among all the objects in the SExtractor catalog. The galaxies we are considering to be fLSBs are not classical dwarf galaxies but the much fainter objects defined in the introduction. We distinguished fLSBs from other object types by applying a series of selection criteria.

- First, we selected objects fainter than $R=21$.

This selection criterion for fLSBs is based on the properties of tidal dwarf galaxies because fLSBs could possibly be tidal dwarfs. Tidal dwarf galaxies have masses between $10^{7}$ and $10^{8} M_{\odot}$ (Bournaud et al. 2003). Assuming a $M / L$ ratio in $R$ of about 5 (Mateo 1998) for the most massive dwarf galaxies, their apparent magnitude should be fainter than $R \sim 21$ at the Coma cluster redshift. This defines the brightest magnitude cut for fLSB selection. It is also in good agreement with the LSB selection criteria used in U96. 
- Second, we identified fLSBs according to the shape of their surface brightness profile.

We differentiated fLSBs from other faint objects using Gaussian radial surface brightness profiles. Although fLSBs typically have exponential surface brightness profiles, U96 found that fLSB selection based on exponential profiles generates a large number of false candidates in the rich environment of Coma, due to the proximity of neighboring objects. In our data, exponential fits are good when confined to the inner regions of the galaxies, but because Gaussian fits are less sensitive to crowding they give better fits up to the outer regions of the galaxies. Instead of using exponential profiles, U96 proposed to select fLSBs by $\chi^{2}$-fitting of Gaussian curves to the radial surface brightness profiles of fLSBs. As shown in Sect. 2.4, this does not mean that an exponential is not the proper form of fLSB profile. Rather, the Gaussian profile is the result of the intrinsic (exponential) shape convolved with instrumental effects (PSF, seeing). Then, following U96, we used Gaussian fits to carry out the initial fLSB selection.

We fit a Gaussian form plus a constant background to the linear-scale surface brightness profiles on the $R$ image. Initially, we let the radial profiles extend to a radius $\theta_{\max }=$ 2.5 arcsec from the center of each object, which, as determined by visual inspection, encompasses the entire range of fLSB sizes.

- Third, we selected initial fLSB candidates with radius greater than 0.6 arcsec and $R$ central surface brightness fainter than $\mu_{\mathrm{R}}=24 \mathrm{mag} \operatorname{arcsec}^{-2}$. The size threshold was chosen above the seeing radius in order to limit contamination by globular clusters which at the distance of Coma, appear as point sources.

- Fourth, we optimized the fit parameters for all initial candidates and selected all candidates with acceptable fits.

We optimized the final value of $\theta_{\max }$ for all the selected candidates $(\sim 1100)$ to ensure that none of their surface brightness profiles were contaminated by surrounding objects. The optimized $\theta_{\max }$ for each candidate was determined by visual inspection. The fitting procedure was repeated. After inspecting all candidates visually we selected as final fLSBs all the candidates that yielded an acceptable (the probability of finding a larger $\chi^{2}$ value is smaller than $10 \%$ ) Gaussian fit to a distance of $\theta_{\max }$. The resulting sample contains $735 \mathrm{fLSBs}$.

After selecting the fLSB sample, we checked that their inclinations did not introduce any bias in the selection procedure. If fLSBs are disk-like, we might expect highly inclined fLSBs to have their surface brightness artificially increased, making their detection easier. However, we checked that high central surface brightness objects are not systematically highly elliptical. The fLSBs with the faintest central surface brightnesses have an ellipticity $8 \%$ smaller than the fLSBs with the brightest central surface brightnesses. Since this is smaller than the uncertainty in the magnitudes, this effect is negligible.

In what follows, we will refer to $\sigma$, the standard deviation of the Gaussian fit, as the fLSB radius except when explicitly noted. Note that the final values of $\sigma$ and $R$ central surface brightness changed from our initial cutoffs because of the optimization on $\theta_{\max }$ performed after the initial candidate selection. For example, when $\theta_{\max }$ was diminished in order to avoid pollution by neighboring objects, the fit value of $\sigma$ changed because the brightness profile was also modified when the neighbor was removed. Nevertheless, Figs. 1 and 2 show that most of the fLSBs still fall within the original selection criteria.

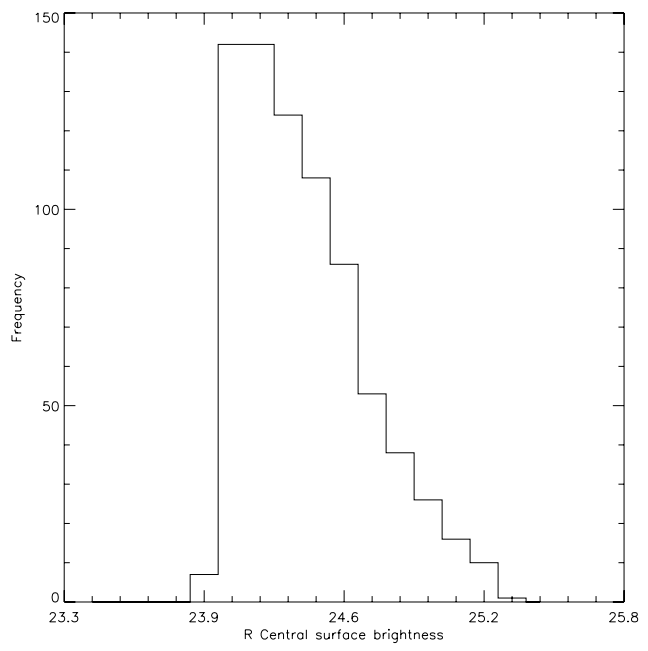

Fig. 1. Histogram of the central surface brightness values of the fLSBs.

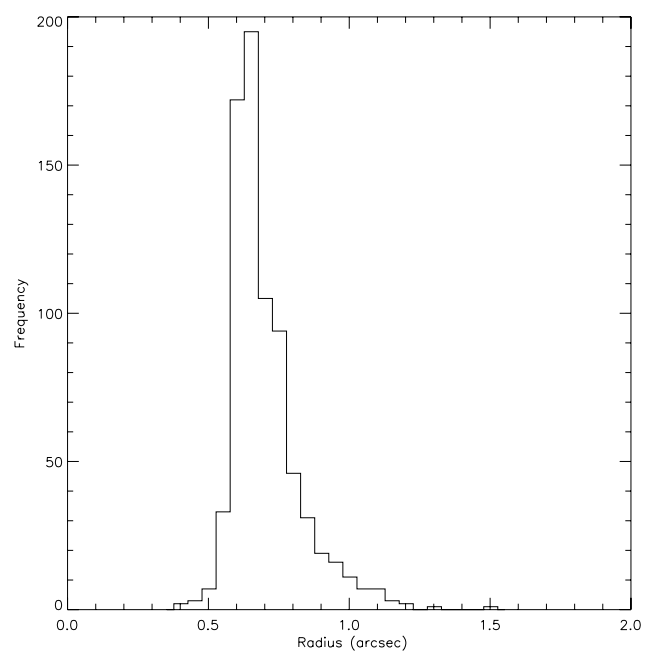

Fig. 2. Histogram of the radius of all the selected fLSBs.

\subsection{Point Spread Function effects on $f L S B$ profiles}

As mentioned in the previous section, fLSBs typically should have exponential profiles. However, Gaussian profiles provide a better fit to the data. This is due to seeing effects. To demonstrate this, we first convolved an exponential profile with scale length of 1.4 arcsec (the mean scale factor from U96) with the average Gaussian fits of the point spread function (PSF) in our data. We found the net result to have a shape that is better fit by a Gaussian than an exponential (see also Fig. 5).

We further investigated the effects of seeing variations on an exponential profile across the field of view. To quantify the instrumental effects on the PSF across the Coma field, we derived the seeing in 100 sub-regions by fitting 2D elliptical Gaussian profiles on $\sim 800$ known stars between magnitudes $I=18.5$ (to avoid saturation) and $I=20.5$ (to avoid confusion with compact galaxies, see A06). We then computed the orientation of the major axis of the stars and the $F W H M$ along the major and minor axes. This was done on the same $R$ band images used for the fLSB detection.

We then computed and smoothed these maps to produce Figs. 3 and 4. These maps show the average of these PSFs, and the ratio between the $F W H M$ of the minor and major axes. We also averaged the orientation of the major axis. We carried out 

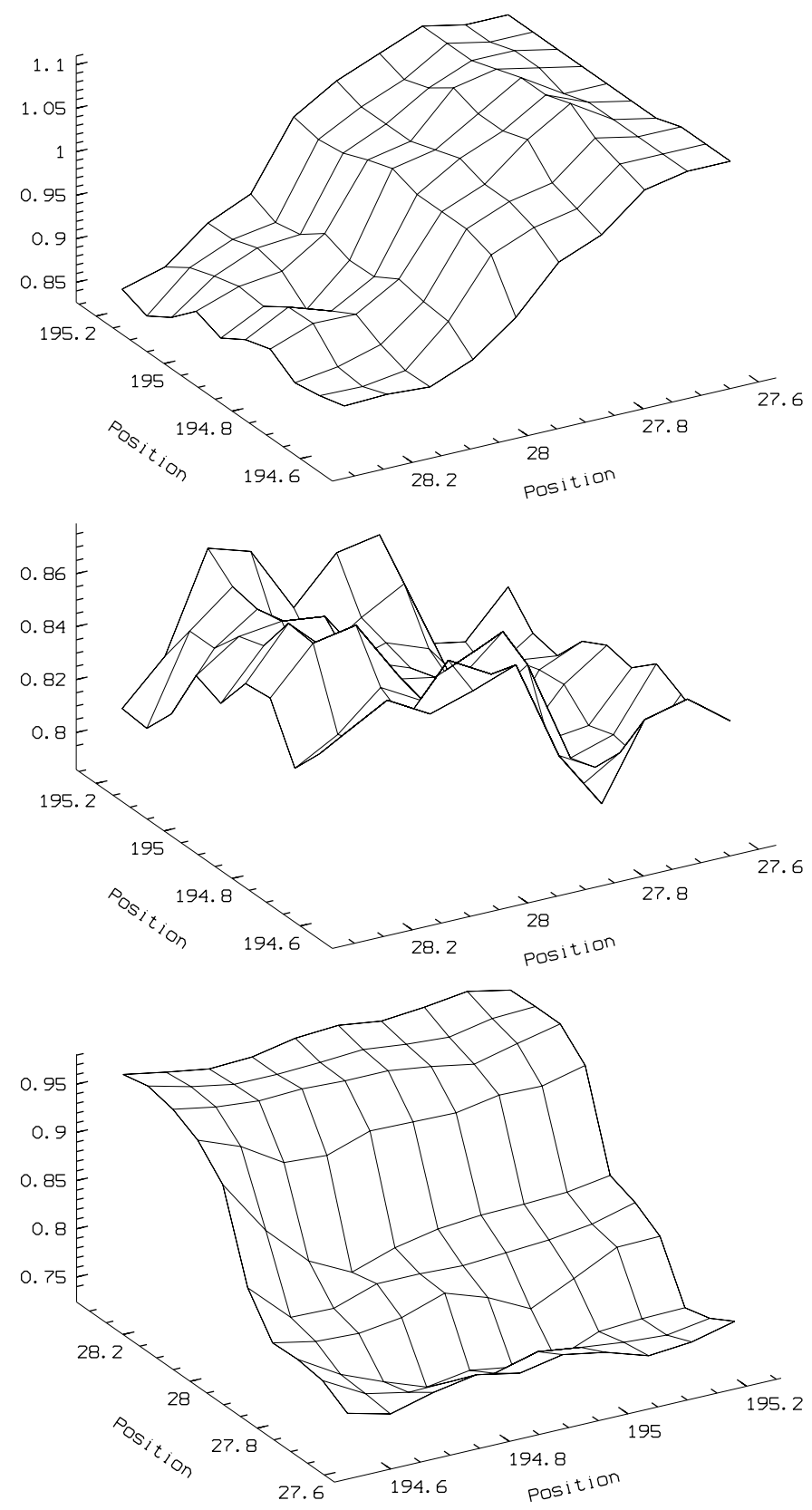

Fig. 3. 3D representation of the PSF FWHM along the major axis (top) and minor axis (middle), and 3D representation of the ratio of the minor axis $F W H M$ to the major axis FWHM (bottom). $\alpha$ and $\delta$ are given in decimal degrees.

the averaging over each artificial pixel $\left(\sim 0.07^{\circ} \times 0.08^{\circ}\right.$ in size $)$, using an adaptive kernel technique (e.g. Adami et al. 1998).

We clearly see on these maps an elongation of the point spread fucntion (PSF) along the $\delta$-direction (except in the northeast area). It is larger in the southern region ( $F W H M$ close to $1.05 \mathrm{arcsec})$ than in the north $(F W H M$ close to $0.9 \operatorname{arcsec})$ while the minor axis is relatively constant (FWHM close to $0.83 \mathrm{arcsec}$ ) on the whole field. This results in a major/minor axis ratio of more than 0.9 in the north and less than 0.8 in the south, perhaps partially due to bleeding in the CCD readout.

To examine the effect of such a PSF on the observed profiles, we convolved an exponential profile using the mean scale factor from U96 (a scale of 1.4 arcsec) with the average Gaussian fits of the PSF in our data.

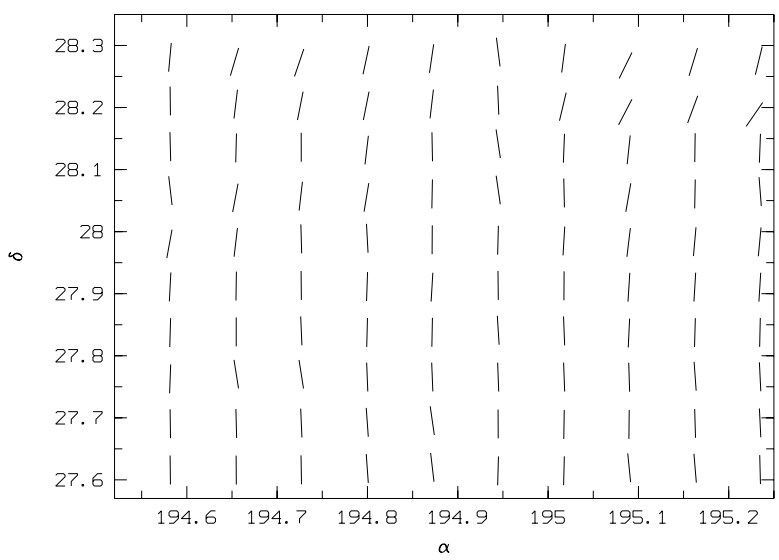

Fig. 4. Map of the major axis orientation. $\alpha$ and $\delta$ are given in decimal degrees (note that $\alpha$ increases to the right).

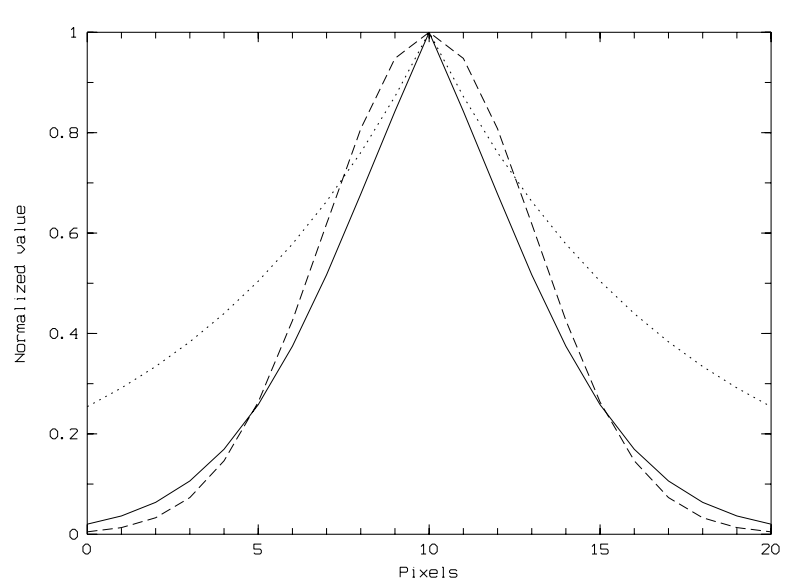

Fig. 5. Plots for the whole Coma cluster region. Small-dotted line: original exponential profile. Continuous line: original exponential profile convolved by the PSF. Dashed line: mean fLSB profile observed in our data. All curves have been normalized to the same maximal value. Pixels are 0.205 arcsec.

The results shown in Fig. 5 imply that the convolved profile is well fit by a Gaussian and we also checked that the difference in PSF between the north and south regions only has a minor effect. This justifies the use of a Gaussian fit to find the fLSBs with no necessity to treat the north regions differently from the south.

\subsection{Step 3: colors and total magnitudes}

In Step 3 we computed the total magnitudes and colors of the 735 fLSBs we detected in Step 2. Total magnitudes were calculated from the radial surface brightness profiles while colors were derived from common aperture photometry.

We calculated total magnitudes by integrating to infinity the Gaussian radial surface brightness profiles generated in the previous step and converting the total counts to magnitudes. Following the discussion in Sect. 2.4, we used the Gaussian profiles rather than the exponential profiles because the Gaussian profiles describe the observed data more accurately. The central surface brightness values were derived by extrapolating the Gaussian fits. Inspection of the profiles in Fig. 6 suggests that the central surface brightness values are reliable. 

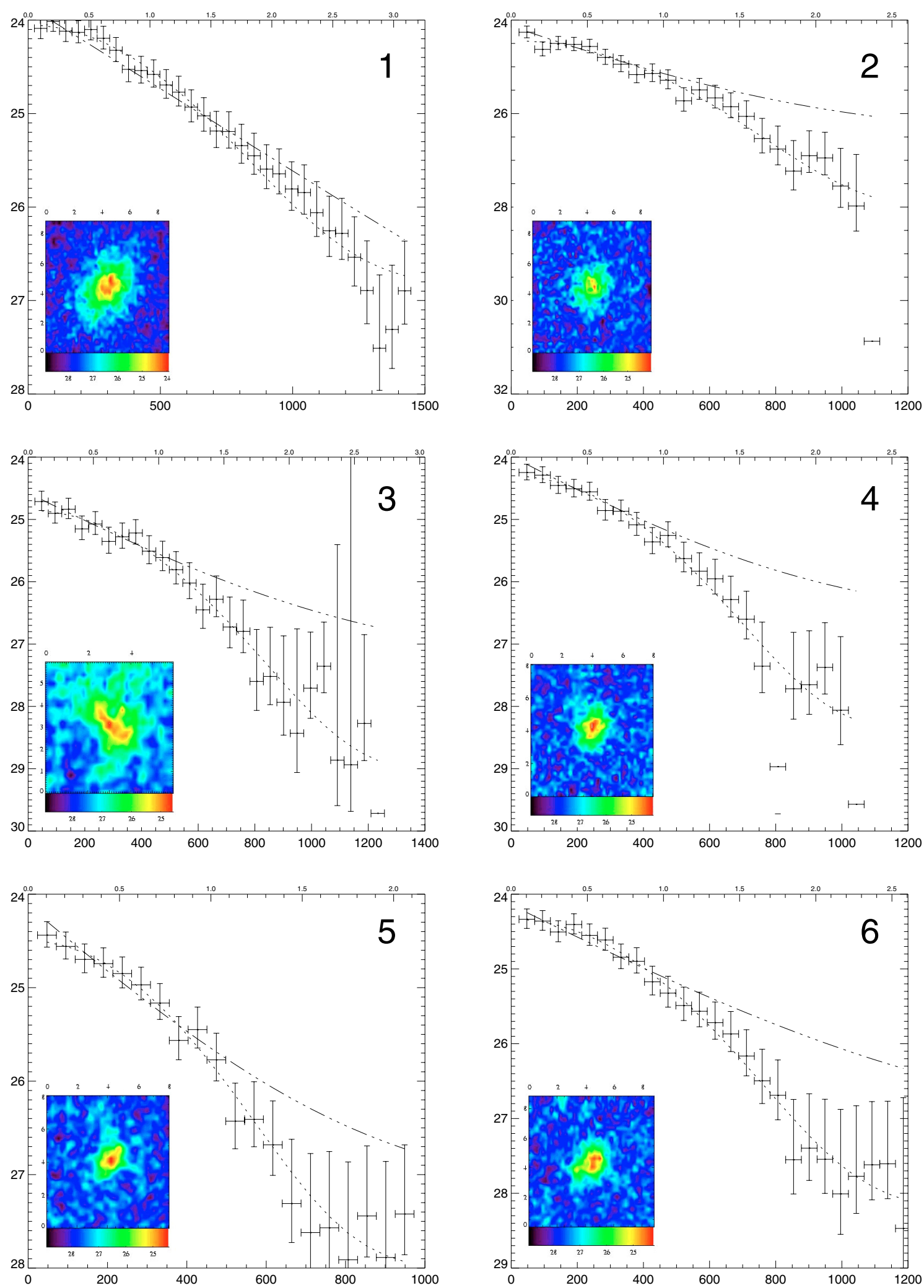

Fig. 6. Six sample plots showing the $R$ radial intensity profiles of the fLSBs we detected, along with the best Gaussian (dotted curve) and exponential (dashed curve) fits. The bottom $X$-axes are in pc. The top $X$-axes are in arc seconds. The $Y$-axes are in $R$ mag per square arc seconds. The exponential fits show some curvature because these fits (as well as the Gaussian model) include a term for the background.

We measured colors by determining $B$ and $R$ magnitudes for each fLSB within the same aperture. The aperture size was adapted to the radius and crowding of each fLSB. The aperture radius was taken to be the final $\theta_{\max }$ (see Sect. 2.2), which was also the inner radius of the local sky background annulus.
The outer radius of the local sky background annulus was set to 4.5 arcsec, except in case of overlapping neighboring objects. When close neighbors were present, the outer radius of the local sky was set to the maximum possible radius free of other objects detectable above the background. 
Table 1. Column 1: fLSB identification numbers; Cols. 2-3: RA and Dec (equinox 2000); Cols. 4-5: total magnitude in $R$ and $B$ based on integrating the Gaussian fits to infinity for the $R$ and $B$ images; Col. 6: $B-R$ color derived using a fixed aperture photometry; Cols. 7-8: central surface brightness for $R$ and $B$ derived by interpolating the best Gaussian fits to $r=0$; Cols. 9-10: radius in arc seconds for $R$ and $B$. This table only shows the first $5 \mathrm{fLSBs}$, the full table can be downloaded from http://www. astro.northwestern.edu/ ulmer/private/coma/lsb-table.txt or http://cencosw. oamp. fr/.

\begin{tabular}{llllllllll}
\hline \hline ID & RA & Dec & $M(R)$ & $M(B)$ & $B-R$ & $\mu_{0}(R)$ & $\mu_{0}(B)$ & $\sigma(R)$ & $\sigma(B)$ \\
\hline 1 & $12: 58: 30.59$ & $28: 22: 56.70$ & 22.90 & 23.75 & 0.89 & 24.19 & 25.30 & 0.72 & 0.88 \\
2 & $12: 58: 10.47$ & $28: 22: 51.30$ & 22.70 & 24.05 & 1.37 & 24.08 & 24.95 & 0.77 & 0.46 \\
3 & $12: 58: 16.78$ & $28: 19: 46.80$ & 23.09 & 24.22 & 1.15 & 24.27 & 25.49 & 0.59 & 0.74 \\
4 & $12: 58: 13.19$ & $28: 19: 9.70$ & 22.89 & 23.74 & 0.84 & 24.38 & 25.32 & 0.75 & 0.80 \\
5 & $12: 58: 28.60$ & $28: 19: 7.50$ & 22.81 & 24.00 & 1.29 & 24.18 & 25.02 & 0.78 & 0.34 \\
\hline
\end{tabular}

\subsection{Total magnitude error estimates}

We investigated magnitude uncertainties by comparing the magnitudes of fLSBs detected twice. There is a $\sim 7$ arcmin overlap band between the two sets of images acquired to cover the entire cluster field. For fLSBs detected in both image sets, the total magnitude differences between both detections are less than $0.3 \mathrm{mag}$, in good agreement with the uncertainties found by A06.

Typical uncertainties for the colors are $0.35 \mathrm{mag}$ at $R \sim 24$ and 0.15 at $R \sim 20$ (estimated from the quadratic sum of individual magnitude uncertainties given in A06).

\subsection{Comparison with SExtractor total magnitudes}

Figure 7 shows that, for the very peculiar object class investigated here, SExtractor total magnitude estimates can differ from the analysis used to derive magnitudes of fLSBs, especially for the brightest fLSBs (contrary to normal galaxies in Coma: e.g. A06). We also found the same behavior in the $B$ band data. The systematic offset is possibly due to a source confusion effect as the brighter fLSBs are also larger. Given the uncertainties in the derived magnitudes, however, the difference between the SExtractor magnitudes and ours does not strongly influence our conclusions.

\subsection{The Coma field $f L S B$ sample: summary}

We found 735 faint, low surface brightness galaxies in the direction of the Coma cluster with central surface brightnesses ranging from $\sim 24$ to $25.5 R$ mag $\operatorname{arcsec}^{-2}$ (except for $10 \mathrm{fLSBs}$ which are brighter) and from 24 to $27 B$ mag $\operatorname{arcsec}^{-2}$. The total magnitudes range from $\sim 21 \leq R \leq 24.5$ and $\sim 22 \leq B \leq 26$. The colors are distributed for most fLSBs between $0.2 \leq B-R \leq 2.6$ and peak at 1.2. fLSB radii range in $\sim 0.4 \leq \sigma \leq 1.5$ arcsec corresponding to $\sim 0.17 \leq \sigma \leq 0.66 \mathrm{kpc}$ but the majority have radii between $\sim 0.6 \leq \sigma \leq 0.8$ arcsec or $\sim 0.26 \leq \sigma \leq 0.35 \mathrm{kpc}$. After quadratically subtracting the seeing radius value to these numbers, most of the fLSBs fall in the range $[0.20,0.31] \mathrm{kpc}$. In contrast, globular clusters have a half light radius (with a partially different radius definition) of $\sim 0.003 \mathrm{kpc}$ (e.g. van den Bergh et al. 1991; or Jordán et al. 2005).

Although the fLSBs we detected along the Coma line of sight are very small and faint, they are certainly not spurious since all were found on the $R$ images but also on the $B$ images. Because the SExtractor detection thresholds in $R$ were quite stringent (detection threshold of $2 \sigma$ and minimum number of pixels of 9 above threshold (see A06)), a number of fLSBs were therefore missed in $R$, but the detected ones were quite obvious and then also detectable in other bands.

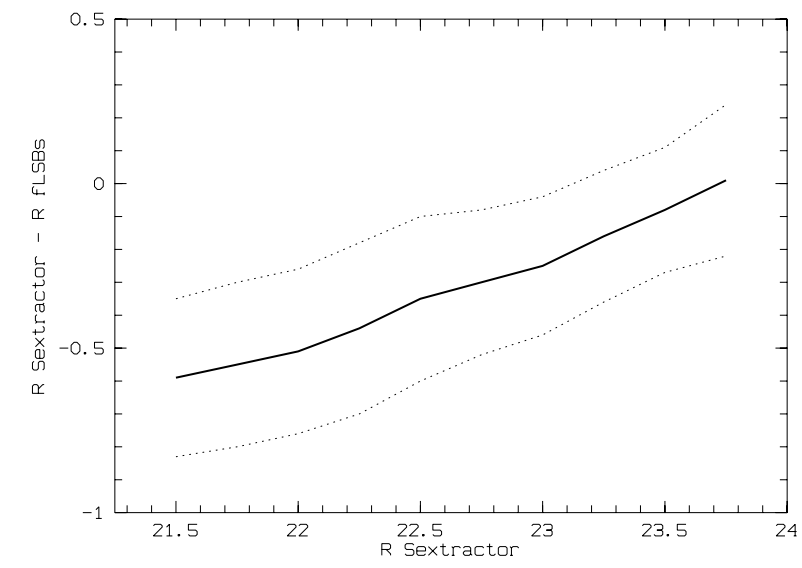

Fig. 7. Thick line: mean difference between SExtractor total $R$ magnitudes and present integrated magnitudes versus SExtractor total magnitudes for the $R$ band. Dotted lines: $1 \sigma$ error envelope.

Our fLSBs are similar in terms of size and total brightness to those found in other clusters by the most recent fLSB searches. They most closely resemble those found in Virgo by Sabatini et al. (2005) with central surface brightnesses of $B \sim 26 \operatorname{arcsec}^{-2}$ and absolute $B$ mag of about -10 , and those found in Ursa Major by Roberts et al. (2004) with a central surface brightness average of $24.5 \mathrm{mag} \operatorname{arcsec}^{-2}$ and scale lengths between 0.23 and $0.35 \mathrm{kpc}$. The fLSB colors are also in good agreement with the expectations from Conselice et al. (2003) who find, in the Perseus cluster, $B-R$ colors ranging from 0.7 to 1.9 , with a mean of 1.15 for galaxies as faint $R \sim 21.3$ at the Coma cluster redshift. A small portion of the catalog of our results is given in Table 1 where the web page address to the full catalog is given.

\subsection{Coma cluster membership}

We used two methods to investigate the cluster membership of our fLSB sample: a statistical comparison with an empty field and an absolute magnitude versus central surface brightness comparison.

In order to put on a firmer ground the Coma membership of our fLSBs, we need to estimate the number of foreground and background galaxies satisfying our fLSB selection criteria. We therefore applied our fLSB selection procedure to a $30 \times 30 \operatorname{arcmin}^{2}$ empty field extracted from the F02 field in the deep VVDS $R$ imaging survey (McCracken et al. 2003), observed with the same instrument (CFH12K), the same $R$ filter, and free from nearby rich structures (VVDS collaboration, private communication). The seeing values of these images are also similar to the seeing for Coma: $0.8 \operatorname{arcsec}$ in $R$ and $0.9 \operatorname{arcsec}$ in $B$. 

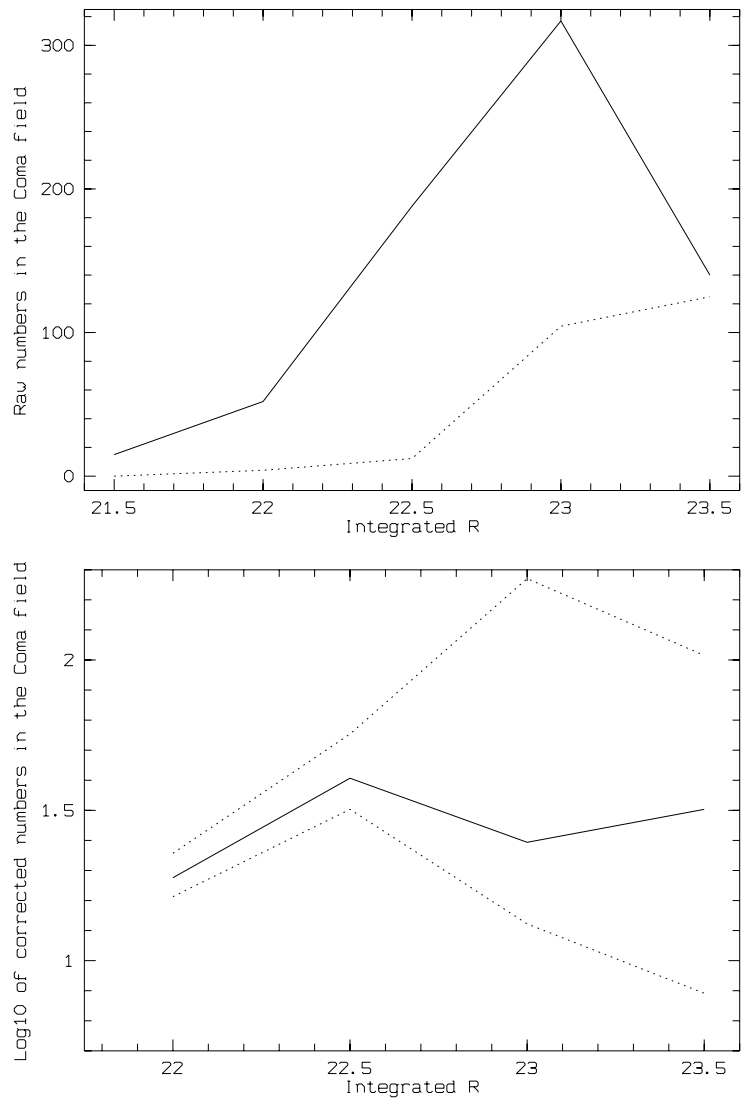

Fig. 8. Upper figure: without detection efficiency corrections: the solid line is the raw number of fLSBs detected along the Coma cluster line of sight; the dotted line is the raw number of fLSBs detected along the empty field line of sight scaled to the Coma cluster field size. Lower figure: corrected for detection efficiency: $\log 10$ of the ratio between fLSBs detected in the Coma cluster field and fLSBs detected in the empty field scaled to the Coma cluster field size. The continuous line is the mean value, the dotted lines are the ratios that delimit $\pm 10 \%$ uncertainty in the detection efficiency estimates.

We corrected for detection efficiencies using McCracken et al. (2003) for the VVDS $R$ data and A06 for the Coma $R$ data. This is shown in Fig. 8. We found that the number of field galaxies satisfying the fLSB selection criteria are less than $4 \%$ of the total number of fLSBs detected along the Coma line of sight.

Another way to discriminate between cluster and line of sight fLSBs is the absolute vs. surface brightness relationship. In their work on the Perseus cluster, Conselice et al. (2002, see their Fig. 6) have shown that the locations of background and cluster galaxies in the absolute magnitude vs. surface brightness plane are very different. We placed our Coma line of sight and field fLSBs on a similar plot (Fig. 9), which clearly shows that the location of most of our fLSBs is inconsistent with the location of the empty field fLSBs. Only $5 \%$ of fLSBs on the Coma line of sight overlap with the empty field location, which is in good agreement with our previous estimate and with the work by Conselice et al. (2002).

These two methods show that the large majority of our fLSBs are likely Coma members and the foreground and background fLSBs are only a minor contribution to the Coma sample.

\section{Comparison with other surveys}

To illustrate the sensitivity of our survey, we compare our sample with previous fLSB catalogs in the core of the Coma

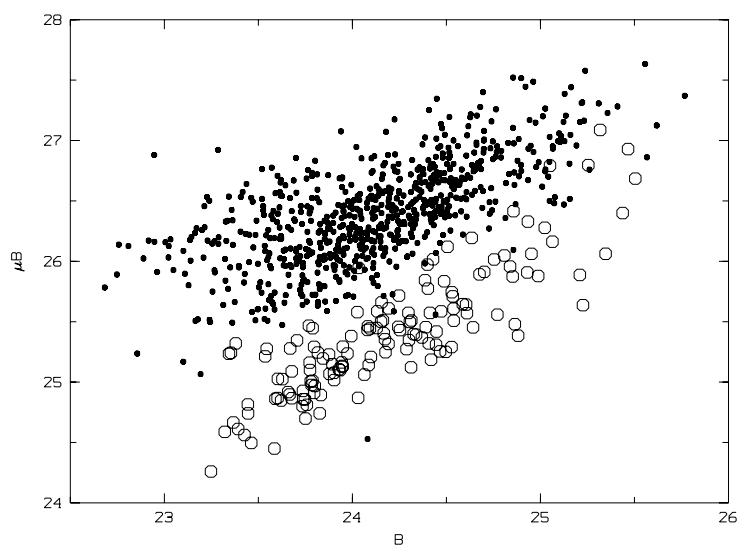

Fig. 9. $B$ magnitude versus $B$ central surface brightness. Small dots: Coma line of sight fLSBs, open circles: empty field fLSBs.

cluster (U96) and with the catalog of low surface brightness galaxies in Fornax (Bothun et al. 1991).

\subsection{The Coma cluster core survey}

U96 conducted a survey of low surface brightness galaxies in the core of the Coma cluster. The area they surveyed $(\sim 7.5 \times$ $7.5 \mathrm{arcmin}^{2}$ ) is centered just South of the dominant galaxies and corresponds to portions of two CCDs in our southern image. The catalog used by U96 to select fLSBs is currently one of the deepest surveys of the Coma cluster with a completeness of $50 \%$ down to $R=25.5$, but with a seeing close to 1.4 arcsec. For comparison, we applied our selection criteria to the U96 detections (see Figs. 1 and 2) in terms of surface brightness and minimal object size. We also limited the U96 sample to the magnitude range $21<R<22.5$ as, at the upper limit, our fLSB detections are $50 \%$ complete (see A06). We should then expect to recover statistically in our sample about $50 \%$ of the 7 U96 fLSBs selected. We did recover $3 \mathrm{fLSBs}$ in our data, a value very close to the $50 \%$ expected level.

\subsection{Comparison with the Fornax cluster}

We also compared our fLSBs with the Fornax sample of Bothun et al. (1991). The curved lines drawn on Fig. 10 are derived by assuming an exponential profile for the galaxies with different scale lengths as indicated by the diagonal dashed lines ( $\alpha$ being the exponential scale factor in arcsec). The region right of each curved solid line (toward lower $B$ ) is where we would expect to detect fLSBs for each sample, given the angular diameter (smaller objects cannot be distinguished from stars) and isophotal surface brightness limits (the faintest level out to which an image is actually detected) noted next to these curves. As expected, this figure shows that most of our fLSBs fall to the right of the left-most curved line. If we correct for the distance of Fornax, the brighter end of our fLSBs overlaps with the faint end of the Fornax dwarfs. This demonstrates that our objects are similar to the Fornax objects.

\section{4. fLSB color analysis}

\subsection{Colors as a function of $f L S B$ size}

In this section we examine how the fLSB colors vary with the radius $\sigma$ (Fig. 11). The smaller fLSBs have a large color scatter 


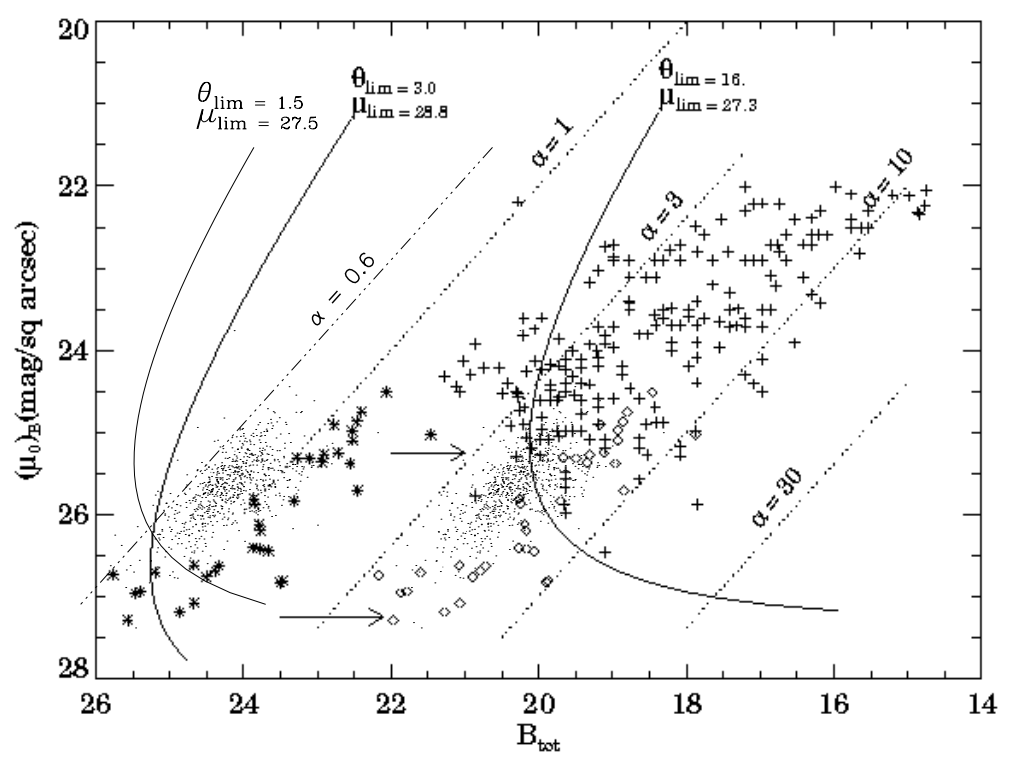

Fig. 10. Figure reproduced from U96 with the addition of our data: central surface brightness as a function of total magnitude in the $B$ band. The pluses indicate Fornax galaxies from Bothun et al. (1991), the asterisks are from U96, the diamonds are the U96 fLSBs put at the distance of Fornax (as denoted by the arrows), the dots to the left are our fLSBs and the dots to the right are our fLSBs put at the distance of Fornax. The solid curves show the selection function that relates the limiting central surface brightnesses and the limiting diameters of the objects to the exponential scale factor given by $B_{\mathrm{tot}}=-0.6689+5 \log \left[\left(\mu_{\lim }-\mu_{0}\right) / \theta_{\lim }\right]+\mu_{0}$ where $\mu_{\lim }$ is the limiting isophotal magnitude, $\mu_{0}$ is the observed central surface brightness and $\theta_{\lim }$ is the limiting diameter in arc seconds. These values are only an estimate. The values $\alpha$ are the exponential scale factor in arcsec.

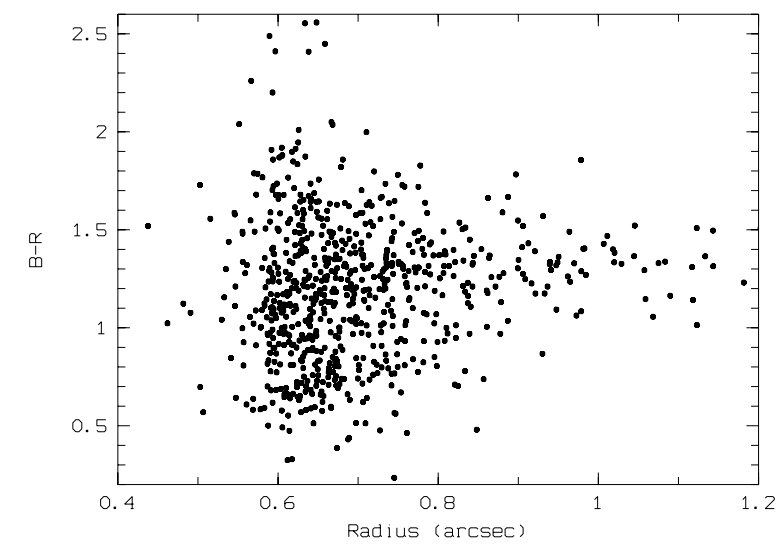

Fig. 11. $B-R$ color vs. fLSB radius.

while the larger fLSBs have a better-defined color sequence centered around $B-R \sim 1.3$. We could expect such a wider spread of colors for the smaller fLSBs since their lower binding energies mean they are more likely to be affected by the cluster environment (Grebel 2001) or more likely to lose metals produced by supernovae (e.g. Kodama \& Arimoto 1997). Alternatively, there could also be more line of sight contamination toward the faint end of our sample, since the number of line of sight objects increases with magnitude. The color scatter of fLSBs is further investigated with the color-magnitude relation in the following sections.

\subsection{Color-magnitude relation}

One of the best known relations between the global properties of galaxies and their stellar populations is the color-magnitude relation (CMR). Luminous early type galaxies in clusters are observed to be redder than fainter ones. This progressive reddening of elliptical galaxies with increasing luminosity is known as the CMR red-sequence. The slope seen in the CMR redsequence is driven primarily by a luminosity-metallicity correlation (e.g. Kodama \& Arimoto 1997; or Vazdekis et al. 2001): brighter galaxies have greater binding energies and can therefore become more metal-rich and thus redder than fainter ones. The CMR red-sequence for the bright galaxies in the Coma cluster has been well studied (e.g. Terlevich et al. 2001; Odell et al. 2002; López-Cruz et al. 2004) and can be compared to our fLSBs.

López-Cruz et al. (2004) found the best fitting CMR redsequence for Coma early type galaxies to be:

$B-R=-0.046 R+2.22$.

This relation is consistent with most literature studies (e.g. Adami et al. 2000, 2006) and, for comparison with our data, was corrected for our specific $B$ and $R$ filters using the transformations given by Fukugita et al. (1995). Results are shown in Fig. 12 along with the color magnitude diagram of our fLSBs. Surprisingly, the fLSB colors are centered on the given CMR red-sequence for objects up to $10 \mathrm{mag}$ fainter than the brightest Coma elliptical galaxies! López-Cruz et al. (2004) found a very narrow ( 0.06 mag Gaussian deviation) CMR red-sequence for the bright objects. Similarly, we fit a Gaussian to the fLSB color distribution and corrected our results for the intrinsic uncertainty on the magnitudes $(\sim 0.25$ at $R=23$, A06). We then find an intrinsic scatter of 0.27 mag around the López-Cruz et al. (2004) CMR red-sequence, clearly larger than for the bright objects but still significantly lower than for the whole object distribution (Fig. 12) which shows a 0.73 intrinsic dispersion in the $B-R$ $[21,24.5]$ range. A Kolmogorov-Smirnov test performed on the $R / B-R$ relation shows that the two populations (the whole sample and the fLSB sample) are different at the $99.99 \%$ level and confirms that our fLSBs are not a randomly selected sample 
from the whole line of sight object population. We note that the star contribution to the object counts in that magnitude range is smaller than $10 \%$ and therefore negligible (A06; Bernstein et al. 1995).

For discussion, we can define three regions in our color magnitude diagram: the sequence of all the fLSBs within one standard deviation of the main CMR red-sequence, the red population above, and the blue population below 1 standard deviation (Fig. 12).

\subsection{Colors of $\mathrm{fLSBs}$ and empirical templates}

Empirical color templates can be used to investigate the nature of our fLSBs. The empirical templates of Coleman et al. (1980) predict $B-R$ colors (in the exact filters we use) of 1.62 for elliptical galaxies, 1.11 for Sbc galaxies, 0.94 for Scd galaxies and 0.7 for irregular galaxies. Fukugita et al. (1995) also predict similar colors, while $B-R$ colors of 0.74 and 0.83 are predicted for the two generic starburst models of Kinney et al. (1996).

In this picture, we confirm that red-sequence fLSBs are indeed E-like objects. Blue fLSBs are probably late type objects, and perhaps even starburst galaxies for the bluest. Red fLSBs are too red to be classical elliptical galaxies. These very red galaxies are as red as the bright Coma galaxies; this should not be the case if they had simply undergone passive evolution, given their low mass and therefore their low binding energy. Field fLSBs are not red enough to explain this red population and peculiar processes in the cluster must be considered, and will be discussed in the following section.

\subsection{Colors of blank-field fLSBs}

Kolmogorov-Smirnov tests on the field and Coma fLSB distributions in the $R / B-R$ space show (Fig. 13) that the two samples are statistically different at almost all magnitudes, except at the Coma fLSB faint end (at $R$ fainter than $\sim 23.65$ ).

The slightly different seeings between the blank-field and the Coma-field only have a minor influence on the computed colors of at most $0.1 \mathrm{mag}$ (the ratios between the $B$ and $R$ seeings are 1.188 in the Coma-field and 1.125 in the blank field).

We have no estimate of the empty field fLSBs distances, so a direct comparison with the Coma fLSBs is impossible but we clearly see that the empty field fLSBs are bluer than the Coma fLSBs at $R$ brighter than $\sim 23.4$. These field galaxies probably have a higher star-forming rate than in Coma.

\section{5. fLSB spatial distribution}

\section{1. fLSB properties vs. location in the cluster}

Mechanisms such as ram pressure, tidal stripping and harassment are greatly increased in denser environments, and if fLSB formation results from one or more of these mechanisms these galaxies should display environment dependent structural properties. We thus expect fLSB structural properties to differ when fLSBs are located in the field, in small galaxy groups, or in rich clusters (Roberts et al. 2004; Sabatini et al. 2005), but also to vary within clusters since galaxy cluster cores are much denser than their outer regions. We first examine the possibility of a simple isotropic relation between fLSB structure and cluster-centric distance. The Coma center is taken here to be the faint galaxy center defined by Biviano et al. (1996), very close to the X-ray center from Neumann et al. (2003).

The $B-R$ colors, $R$ magnitudes, central surface brightnesses and radii of our fLSBs do not show significant dependence with
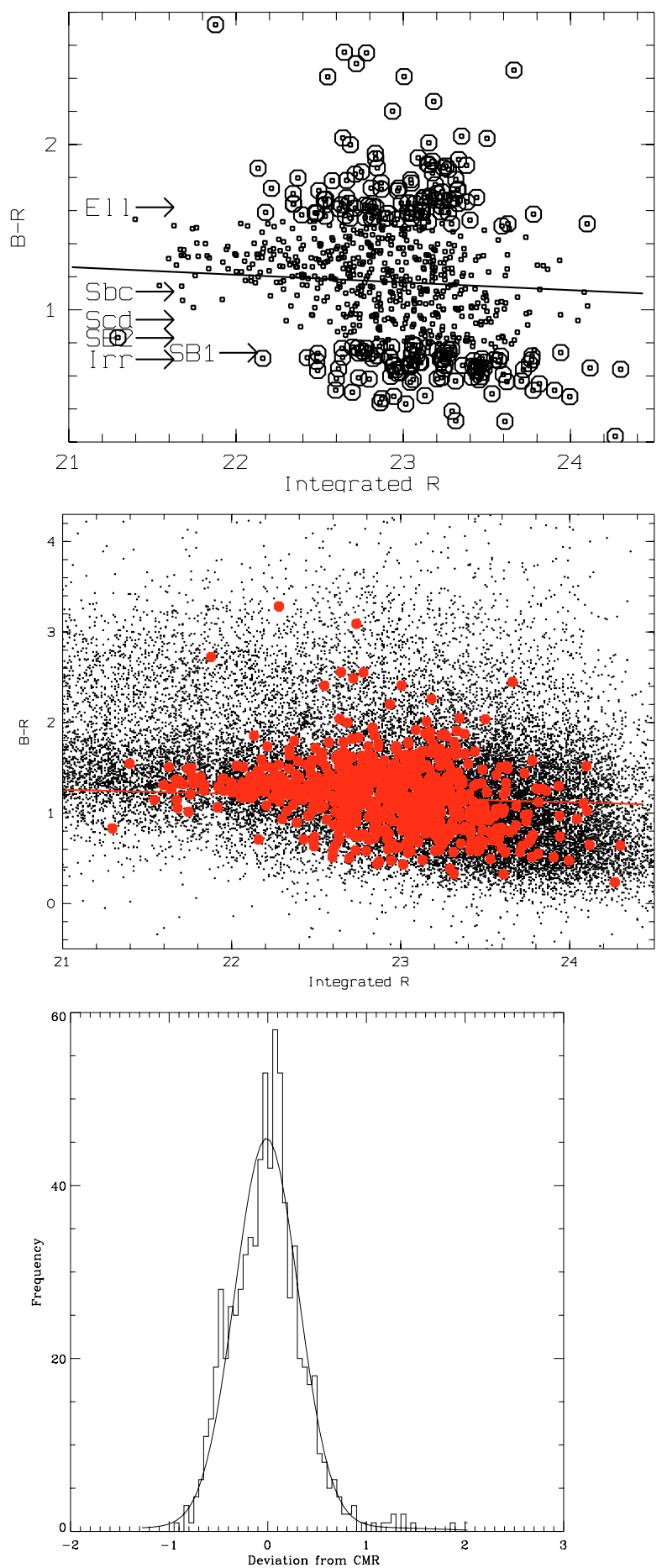

Fig. 12. Upper figure: color-magnitude relation for all the fLSBs along with the CMR red-sequence given by López-Cruz et al. (2004). Circled dots are fLSBs more than 1 standard deviation away from the López-Cruz et al. (2004) relation. Theoretical colors for several synthetic bright galaxy spectrophotometric types are also quoted. Middle figure: fLSB $B-R$ (filled circles) superimposed on the whole object population $B-R$ (small dots). Lower figure: histogram of the deviations around the mean CMR red-sequence given by López-Cruz et al. (2004), along with the best Gaussian fit on this distribution.

distance to the cluster center, in agreement with the result of Sabatini et al. (2005) for fLSB colors in Virgo.

Besides, we can gain further details on how the cluster environment affects fLSB formation by examining the spatial distribution of fLSBs in the cluster. Here we compare 

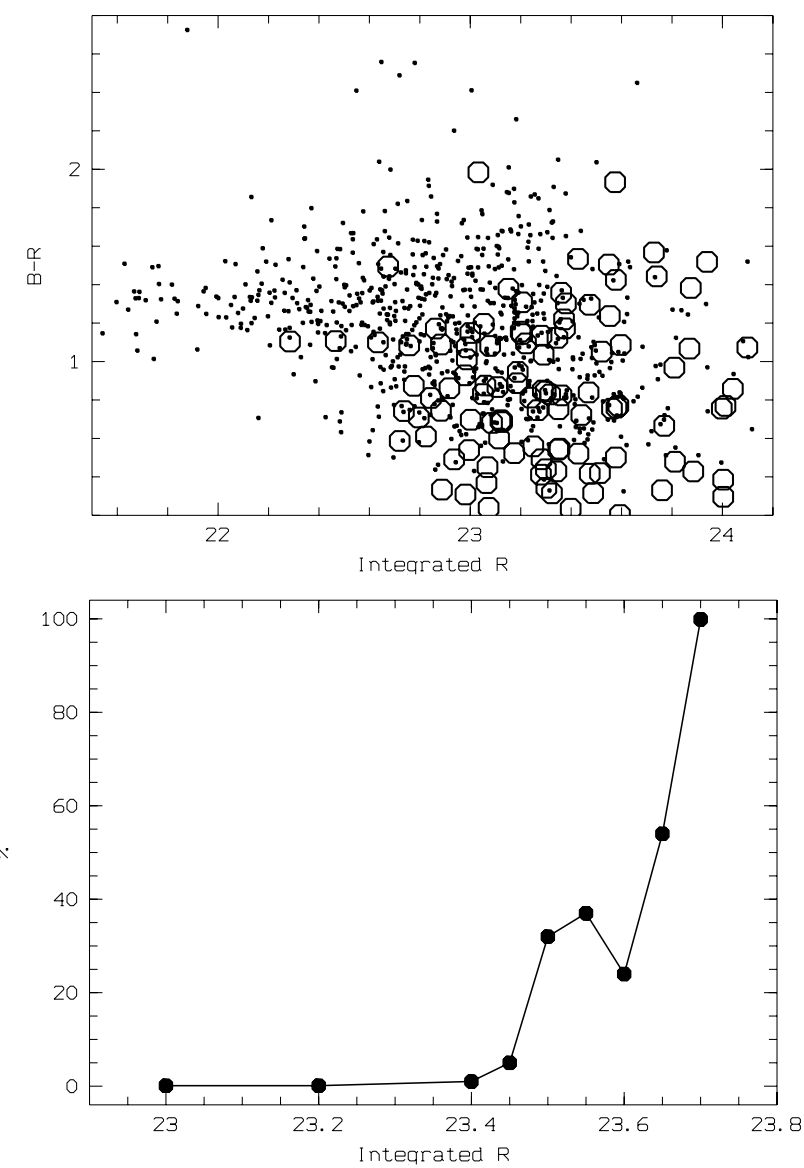

Fig. 13. Upper graph: small dots: fLSBs detected along the Coma cluster line of sight. Large circles: fLSBs detected in the empty field. Lower graph: probability of the Coma and empty field fLSB samples fainter than a given $R$ integrated magnitude to be similar.

our fLSB spatial distribution with the X-ray residuals from Neumann et al. (2003), which indicate potential substructures or in-falling groups. In order to investigate how fLSBs associate with bright galaxies within Coma, we binned our fLSBs in a two dimensional histogram with bin sizes of 1.9 arcmin along right ascension and 2 arcmin along declination. We corrected the counts using the detection efficiencies given in A06. We note that the detection level only varies weakly from east to west across the north and south fields, but changes strongly between the north and south fields.

Then, in order to estimate which fLSB over densities are statistically significant, we divided the fLSB counts by $\sigma_{\text {distribution }}$ (hereafter $\left.\sigma_{\mathrm{d}}\right), \sigma_{\mathrm{d}}$ is the standard deviation of the total number of fLSBs in the region $\alpha=\left[194.9^{\circ}, 195.1^{\circ}\right] ; \delta=\left[27.6^{\circ}, 27.8^{\circ}\right]$, an area free from any peculiar fLSB density peak. The resulting fLSB distribution is shown in Figs. 14 and 15 for all fLSBs, the CMR sequence and the blue fLSBs. The red fLSBs do not show very significant density peaks.

\subsection{Contour plots of the whole fLSB sample}

The fLSBs from the whole sample are distributed all over the cluster with several significant peaks (see Fig. 14a). In particular, we detect:

- a large over-density of fLSBs around of NGC 4889 with a south extension;
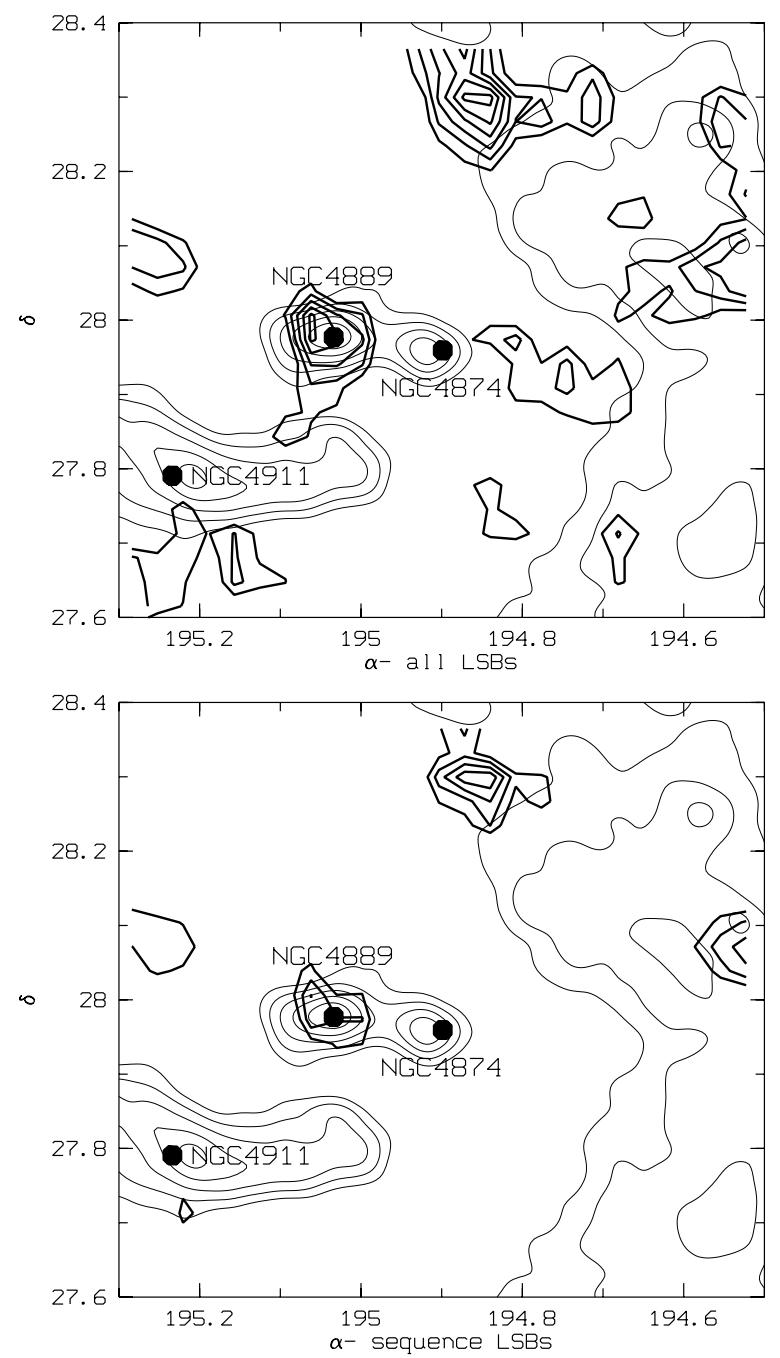

Fig. 14. Contour plots of several fLSB samples (thick contours) superimposed to X-ray residuals from Neumann et al. (2003) (thin contours). Top a): all fLSBs. Bottom b): the CMR sequence fLSBs. The first fLSBs contour in both plots is the $2 \sigma_{\mathrm{d}}$ level and the interval between two levels is $0.5 \sigma_{\mathrm{d}}$ (see definition of $\sigma_{\mathrm{d}}$ in Sect. 5.2). NGC 4911, NGC 4889 and NGC 4874 are plotted as filled dots.

- a possible over-density $\sim 0.22^{\circ}$ north-east of NGC 4889 , at the border of the field;

- an over-density $\sim 0.1^{\circ}$ south of NGC 4911;

- an over-density $\sim 0.1^{\circ}$ west of NGC 4874 ;

- a strong peak north of NGC 4874 at the border of the field. This peak does not appear to be associated with a specific bright galaxy; it could be overestimated, because the fLSB detection level in that region is quite low;

- several peaks coinciding with the west X-ray over density.

The fLSB spatial distribution suggests that some of the Coma fLSBs are associated with the western X-ray over densities. There are over-densities near NGC 4889 significant at the $4.5 \sigma_{\mathrm{d}}$ level but there are no significant over-densities at better than the $2 \sigma_{\mathrm{d}}$ level close to NGC 4874 . This does not mean that there are no fLSBs around NGC 4874, but that their density compared to the whole field is not significantly higher. This suggests that the fLSBs are not directly associated with both giant ellipticals but only with NGC 4889.

The fLSB spatial distribution appears to be anti-correlated with that of large scale diffuse light sources (as defined in 


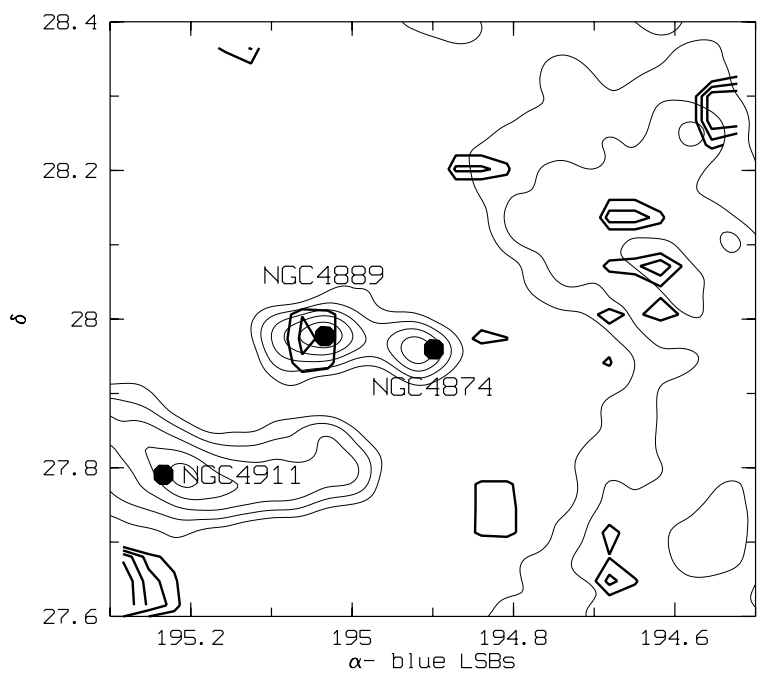

Fig. 15. Same as Fig. 14 for the blue fLSB sample.

Adami et al. 2005a). There are many fLSBs around NGC 4889 but no large scale diffuse light structures, and few fLSBs around NGC 4874 but several large scale diffuse light structures. The diffuse light as defined here is so faint, that the diffuse emission does not explain the lack of fLSBs due to detection efficiency (which has been corrected for, but could in principle still be a problem). This suggests that diffuse light may have formed from the disruption of fLSBs (see also López-Cruz et al. 1997). This hypothesis would agree with the idea that NGC 4874 is the "oldest" giant galaxy in Coma (in the sense that the dominant galaxy NGC 4874 was probably present in the Coma cluster before NGC 4889, cf. Adami et al. 2005b), and therefore fLSBs neighboring NGC 4874 would have had more time to be disrupted by tidal effects and create diffuse light (e.g. Thompson \& Gregory 1993; Lobo et al. 1997).

\subsection{Contour plots of several subsamples}

We now compare the spatial distribution of various subsamples of fLSBs. Figures 14 and 15 show the distribution of fLSBs in the CMR sequence and for blue objects. They exhibit features similar to the full fLSB sample with a few noticeable differences:

- the western groups that show up when we include all fLSBs are generally not present if we only use CMR red-sequence fLSBs, which after detection efficiency corrections, represent $65 \%$ of the sample;

- over-densities are more prominent in the blue fLSBs which represent $22 \%$ of the sample (about twice the red fLSBs); blue fLSBs are distributed throughout the cluster with overdensities near the dominant galaxies, at the field borders, but mainly in the western X-ray extension;

- the red fLSB main over-densities are located away from the center and are not statistically significant $(\geq 2 \sigma)$; however, some of them seem correlated with the western X-ray over densities. After detection efficiency corrections, the red fLSBs represent $13 \%$ of the sample.

\section{Discussion}

For clarity, let us first summarize our main results:

i) we found 735 faint low surface brightness galaxies in the direction of the Coma cluster, consistent with an exponential profile when the PSF is taken into account. The central surface brightnesses range from $\mu_{\mathrm{B}}=24$ to $27 \mathrm{mag} \mathrm{arcsec}^{-2}$, and the total absolute magnitudes from $M_{\mathrm{B}}=-12.9$ to $M_{\mathrm{B}}=-8.9$;

ii) from an empty field comparison, we showed that most of our 735 fLSBs are most probably members of the Coma cluster;

iii) two thirds of fLSBs are consistent with the previously reported CMR red-sequence for bright $(R \leq 18)$ ellipticals in Coma;

iv) we found a statistically significant over density of fLSBs in the core of the cluster around NGC 4889;

v) the fLSBs exhibit no isotropic cluster-centric variations of color, magnitude or central surface brightness, whether we consider them as a whole or red and blue populations separately. However, blue populations (and red ones at a lower level) are preferentially located west of the cluster, coincident with a large X-ray over density.

We stress that this is the first large scale study of objects so faint and difficult to detect in Coma. Recent work as e.g. Andreon \& Cuillandre (2002), Beijersbergen et al. (2002), Iglesias-Páramo et al. (2003), or Lobo et al. (1997) did not use sufficiently deep data to sample efficiently the regime we are studying here. Other work as Trentham (1998) or Bernstein et al. (1995) have too small a spectral coverage or sampled area. In the sections below we discuss our results in terms of several possible origins of the Coma fLSBs.

\subsection{The $C M R$ and $f L S B$ evolution}

Along the results of Secker et al. (1997), Odell et al. (2002) and López-Cruz et al. (2004), we found that the CMR in Coma can be traced similarly for giant ellipticals and low luminosity galaxies represented here by fLSBs. The CMR red-sequence is described by a simple straight line fit down to total magnitudes of $R=24.5$. This effect is extremely interesting because it suggests that galaxies over a range of more than 10 mag have shared a similar evolution process (note that Sandage 1972, had reported a comparable spread over $\sim 8$ mag in Virgo).

The existence of a colour-magnitude relation for cluster galaxies implies that these galaxies are made from uniformly old stellar populations (e.g. Terlevich et al. 2001). Because the CMR is a metallicity effect (e.g. Kodama \& Arimoto 1997), elliptical galaxies experienced an extended period of very efficient star formation at high redshift $(z \geq 2)$ during which the CMR red-sequence was established by successive generations of stars and has been evolving passively since then. Hence the sequence fLSBs in our sample have experienced their major starburst at the same time as the bright ellipticals and have evolved passively since. If we assume that sequence fLSBs are dE galaxies, as shown with color templates, this is consistent with the results of Graham \& Guzmán (2003) who found a continuous structural relation between $\mathrm{dE}$ and $\mathrm{E}$ galaxy classes in Coma.

The formation of red-sequence fLSBs can be explained by a simple collapse/feedback mechanism (Dekel \& Silk 1986). Gas, embedded in dark matter halos present in the universe, collapses under its own gravity to create the first generation of stars. Because the galaxies created in this manner have small masses and low binding potentials, the winds created by the first supernova explosions would eject all the remaining gas out of the galaxy and the metals they produced. This process stops star formation and creates passively evolving fLSBs. 


\subsection{Accretion of sequence $f L S B$ s onto the Coma cluster?}

In the scenario described in the previous section, sequence fLSBs had a formation and evolution similar to the bright cluster galaxies. From the results of Biviano et al. (1996) and Neumann et al. (2003) on the structure of the Coma cluster, we conclude that these fLSBs were formed in smaller galaxy groups (along with the bright galaxies), which later merged to create the Coma cluster as we see it today. However, the CMR sequence fLSBs are not directly clustered around the two dominant galaxies, but only around NGC 4889, while the bright galaxies are still clustered around each dominant galaxy. We can account for this effect by evoking dynamical evolution.

Dynamical friction and relaxation, which only weakly affect low mass galaxies, probably caused the original galaxy groups centered on NGC 4889 and NGC 4874 to evolve in a core-halo structure with the brightest galaxies in their core and the faintest in the halo. The two groups then merged together. Upon accretion onto the Coma cluster, the faint galaxies, which generally have larger velocity dispersions than bright galaxies, were more easily stripped off from the original galaxy group than the bright galaxies in the tightly bound cores. The stripped galaxies were bound to the cluster but were scattered throughout the cluster. If NGC 4874 has been in place at the center of the Coma cluster for a longer time than NGC 4889 (e.g. Neumann et al. 2003), this could explain why the bright galaxies are still clustered around the two giants but the CMR sequence fLSBs are only clustered around NGC 4889. Besides having more time to be stripped, the fLSBs originally around NGC 4874 could have also had more time to be destroyed than those around NGC 4889 (e.g. Thompson \& Gregory 1993). This is consistent with the suggestion by Schombert (1992) that NGC 4874 is a cD galaxy while NGC 4889 is not.

Another scenario is to consider the central region of Coma as the merger of a cluster containing a $\mathrm{cD}$ galaxy and a cluster without a cD, each component then keeping some of its original properties.

\section{3. $f L S B$ to giant ratio}

The fLSBs, however, cannot all be born in groups later accreted into clusters along with the massive galaxies due to the high dwarf to giant ratio found in clusters (e.g. Binggeli et al. 1990; Sabatini et al. 2005). Sabatini et al. (2005, and references therein) have determined that the dwarf to giant galaxy surface density ratio is about 20 in Virgo and 4 in the Local Group. They calculated this ratio by simply dividing the number of galaxies brighter than $M_{\mathrm{B}}<-19$ and those in the range $M_{\mathrm{B}}=[-14,-10]$. Based on this result, they concluded that the dwarfs in Virgo cannot simply have formed (via a standard CDM hierarchical scenario) around giants in the field that fell into the potential well of Virgo. Some dwarf galaxies must have formed in the Virgo cluster. Similarly, Conselice et al. (2003) show that all low mass galaxies in the Perseus cluster cannot originate from simple early collapse. Moore et al. (1998) also suggested there should be an enhancement of dwarfs in clusters with the exception of the very central regions where these galaxies could be destroyed.

To compare with previous work, we computed the fLSB to giant surface density ratio in our data. Because fLSBs are only a subsample of dwarf galaxies, this ratio is an underestimate of the real dwarf to giant ratio. Using the same definition as Sabatini et al. (2005) for Virgo, we found 26 giant galaxies. Among these 26, 13 have a measured redshift and all 13 belong to the Coma cluster. We will assume therefore that all 26 giant galaxies are part of the Coma cluster. The magnitude range in which we detected fLSBs is $M_{\mathrm{B}}=[-12.89,-8.89]$. In order to compare our results with Sabatini et al. (2005), we limited the faint end to -10 and corrected the number of fLSBs by $7 \%$ to take into account the different brightness limits $(-12.89$ versus -14$)$. The $7 \%$ value was estimated using the luminosity function of Bernstein et al. (1995): the galaxies in the magnitude range $M_{\mathrm{B}}=[-12.89,-10]$ account for $93 \%$ of the galaxies in the range $M_{\mathrm{B}}=[-14,-10]$. The resulting number of fLSBs we would have observed in $M_{\mathrm{B}}=[-14,-10]$ is therefore 728 . We then find a ratio of 28 to 1 for all the 728 fLSBs compared to giants. If we assume all are in Coma, the numbers for the fLSB to giant galaxy ratio are comparable to those in Virgo.

It is therefore tempting to conclude, as Sabatini et al. (2005) did for Virgo, that not all the fLSBs in Coma formed around giant galaxies prior to in fall on the Coma cluster. Additional processes, as for example containment of metals in a giant galaxy halo by the intracluster medium, or the formation from the remnants of stripped galaxies are required to explain the higher fLSBs to galaxy ratio.

As shown in Fig. 12, the fLSBs in the blue and red regions may have undergone significantly different evolution processes from the CMR fLSBs. This is related to the fact that the intrinsic fLSB $B-R$ color scatter was clearly larger than for bright galaxies (see Sect. 4.1). If all fLSBs had followed the same evolutionary path, they should all have the same colors (within measurement uncertainties). The large color scatter is probably produced through multiple formation scenarios proposed below.

\subsection{Galaxy harassment and tidal stripping}

The excess of fLSBs in the cluster and their large color scatter can be partly explained if fLSBs are the remnants of normal galaxies, which were transformed into low luminosity objects as they fell into the cluster. Two such scenarios are "galaxy harassment" (Moore et al. 1996) and tidal stripping. Galaxy harassment is defined as frequent high speed galaxy encounters which drive morphological transformations as spiral galaxies move on their orbits across the cluster (Gallagher et al. 2001). Late type galaxies are disturbed by the impulse forces generated by these encounters that strip off mass from the galaxy, drive starbursts and initiate a rapid morphological evolution from large spirals to dwarf ellipticals (Moore et al. 1996). In contrast, tidally stripped galaxies, which can be both spirals and ellipticals, lose stars but do not undergo major morphological evolution. Large ellipticals are simply transformed into dwarf ellipticals. Below we discuss how these two scenarios relate to red and blue fLSB formation.

Blue fLSB could be created from a re-assembly of the outer portions of harassed spirals. One prediction of the galaxy harassment model is that dwarf galaxies should be assembled from the debris tails of the harassed galaxies (Moore et al. 1996; see also Barnes \& Hernquist 1992; Elmegreen et al. 1993; Bournaud et al. 2003; Duc et al. 2004, for galaxy formation scenarios in tidal tails). This process of dwarf galaxy formation would take place in in-falling, spiral-rich groups. The resulting galaxies would be bluer than normal, since built from external spiral parts (that are star forming regions) and significantly fainter. Our blue fLSBs are mostly found along the line of sight of the large $\mathrm{X}$-ray over density west of the cluster. This X-ray over density is probably a collection of galaxy groups in the process of falling into Coma (e.g. Neumann et al. 2003, and references therein) as suggested by the diffuse radio emission in Coma (Giovannini \& Feretti 2002). Because the location of blue fLSBs coincides with 
infalling galaxy groups, the blue fLSBs are likely to have been created from debris of harassed spiral galaxies.

In order to check if this scenario is viable, we compared our results with simulations of tidal dwarf galaxy formation. Such galaxies have masses between $10^{7}$ and $10^{8} M_{\odot}$ (e.g. Bournaud et al. 2003). We estimated the masses of our fLSBs using the $M / L$ ratio of Mateo (1998) translated into the $R$ band, and found that our fLSBs have values of $M / L$ between 5 and 20 ( 5 for the brightest, 20 for the faintest). This corresponds to masses between $3 \times 10^{7}$ and $10^{8} M_{\odot}$, typically in the range predicted by simulations. This shows that the formation of blue fLSBs can occur following the scenario proposed above.

Red fLSBs could be the central remnants of stripped low mass early type galaxies. The metallicity is not uniformly distributed in a galaxy (e.g. Zaritsky et al. 1994). There is a factor between 3 and 5 in metallicity between the central and external parts of elliptical galaxies (Henry \& Worthey 1999). In the tidal stripping scenario, fLSBs would be formed from central metal rich material and be redder than passively evolving galaxies of similar mass. Note that the original elliptical galaxies would have to be already relatively faint in order to create red fLSBs as faint as $R=21$. A similar process was also proposed to explain the creation of red low-mass galaxies in the Perseus cluster (Conselice 2002).

The galaxy harassment and tidal stripping scenarios are in good agreement with the fact that we found the blue (and possibly the red) fLSBs to be correlated with possible infalling groups (including spirals and moderately early type galaxies). These infalling galaxies could have been the source of material used to form the red and blue fLSBs.

\section{Conclusions}

Using a large sample of fLSBs detected along the Coma cluster line of sight, we were able to reach several conclusions regarding their various natures and their origins:

i) From the comparison with an empty field, about $95 \%$ of our detected fLSBs are likely to be part of the Coma cluster.

ii) Two-thirds of the fLSBs (the ones along the CMR sequence) experienced an evolution similar to that of bright ellipticals: they were formed in the same subgroups as the bright galaxies and joined the cluster when the subgroups merged onto Coma. Upon accretion, some fLSBs were ejected from the subgroups, scattered throughout the cluster, but yet retained by the underlying cluster potential. They have been undergoing passive evolution since then. Some could also have been destroyed following the Thompson \& Gregory (1993) scenario. If passive evolution is the explanation for the redsequence fLSBs, the fact that the ratio of red-sequence fLSBs to giant galaxies in Coma is still significantly higher than in the field remains a puzzle. Only numerous fusions of bright galaxies could explain this ratio. We plan to investigate this question in a future paper.

iii) fLSBs that fall on the color magnitude relation are consistent with a simple collapse feedback scenario: fLSBs were formed when gas collapsed and ignited a starburst. Because of their small size and low binding potential, supernova winds could have ejected all the remaining gas and metals from the galaxy and halted additional star formation.

iv) We found indications supporting a scenario in which debris from galaxy harassment can create blue fLSBs.

v) Formation of red fLSBs as the central remnants of small stripped early type galaxies is also a possibility.
Further studies involving redshifts, velocities and gas content measurements are needed to gain more clues on the formation and evolution processes of these fLSBs.

Acknowledgements. The authors thank the referees for useful and constructive comments and are grateful to the CFHT and Terapix teams, and to the French CNRS/PNG for financial support. Some of the authors also acknowledge support from NASA Illinois space grant NGT5-40073, from Northwestern University and from NSF grant AST-0205960.

\section{References}

Adami, C., Mazure, A., Katgert, P., \& Biviano, A. 1998, A\&A, 336, 63

Adami, C., Ulmer, M. P., Durret, F., et al. 2000, A\&A, 353, 930

Adami, C., Slezak, E., Durret, F., et al. 2005a, A\&A, 429, 39

Adami, C., Biviano, A., Durret, F., \& Mazure, A. 2005b, A\&A, 443, 17

Adami, C., Picat, J. P., Savine, C., et al. 2006, A\&A, 451, 1159 (A06)

Andreon, S., \& Cuillandre, J.-C. 2002, ApJ, 569, 144

Babul, A., \& Rees, M. J. 1992, MNRAS, 255, 346

Barnes, J. E., \& Hernquist, L. 1992, Nature, 360, 715

Beijersbergen, M., Hoekstra, H., van Dokkum, P. G., \& van der Hulst, T. 2002, MNRAS, 329, 385

Bernstein, G. M., Nichol, R. C., Tyson, J. A., Ulmer, M. P., \& Wittman D. 1995, AJ, 110, 1507

Bertin, E., \& Arnouts, S. 1996, A\&AS, 117, 393

Binggeli, B., Sandage, A., \& Tammann, G. A. 1985, AJ, 90, 1681

Binggeli, B., Tarenghi, M., \& Sandage, A. 1990, A\&A, 228, 42

Biviano, A. 1998, Proc. Untangling Coma Berenices: A New Vision of an Old Cluster, Marseille, France, June 17-20, 1997, ed. Mazure et al. (Word Scientific Publishing Co Pte Ltd.) and

http://nedwww. ipac. caltech.edu/level5/Biviano/frames.html Biviano, A., Durret, F., Gerbal, D., et al. 1996, A\&A, 311, 95

Bothun, G. D., Impey, C. D., \& Malin, D. F. 1991, ApJ, 376, 404

Bothun, G. D., Schombert, J. M., Impey, C. D., Sprayberry, D., \& McCaugh, S. S. 1993, AJ, 106, 530

Bournaud, F., Duc, P. A., \& Masset, F. 2003, A\&A, 411, L469

Coleman, G. D., Wu, C. C., \& Weedman, D. W. 1980, ApJS, 43, 393

Conselice, C. J. 2002, ApJ, 573, L5

Conselice, C. J., Gallagher, J. S., \& Wyse, R. F. G. 2002, AJ, 123, 2246

Conselice, C. J., Gallagher, J. S., \& Wyse, R. F. G. 2003, AJ, 125, 66

Davies, J. J., Sabatini, S., \& Roberts, S. 2004, PASA, 21, 360

de Blok, W. J. G., McGaugh, S. S., \& Rubin, V. C. 2001, AJ, 122, 2396

Dekel, A., \& Silk, J. 1986, ApJ, 303, 39

Duc, P. A., Bournaud, F., \& Masset, F. 2004, A\&A, 427, 803

Elmegreen, B. G., Kaufman, M., \& Thomasson, M. 1993, ApJ, 412, 90

Feretti, L., \& Neumann, D. 2006, A\&A, 450, L21

Fukugita, M., Shimasaku, K., \& Ichikawa, T. 1995, PASP, 107, 945

Gallagher, J. S., Conselice, C. J., \& Wyse, R. F. G. 2001, Proc. Conf. Dwarf galaxies and their environment, Bad Honnef, Germany, ed. de Boer et al., 213

Giovannini, G., \& Feretti, L. 2002, Proc. Merging Process in Galaxy Clusters, ed. Feretti et al., ASS Library, 272, 197 (Dordrecht: Kluwer Academic Publishers)

Giovannini, G., Feretti, L., Venturi, T., Kim, K.-T., \& Kronberg, P. P. 1993, ApJ, 406, 399

Graham, A. W., \& Guzmán, R. 2003, AJ, 125, 2936

Grebel, E. K. 2001, Proc. Conference Dwarf galaxies and their environment, Bad Honnef, Germany, ed. de Boer et al., 213

Gregg, M. D., \& West, M. J. 1998, Nature, 396, 549

Henry, R. B. C., \& Worthey, G. 1999, PASP, 111, 919

Iglesias-Páramo, J., Boselli, A., Gavazzi, G., Cortese, L., \& Vílchez, J. M. 2003, A\&A, 397, 421

Impey, C., \& Bothun, G. 1997, ARA\&A, 35, 267

Impey, C. D., Sprayberry, D., Irwin, M. J., \& Bothun, G. D. 1996, ApJS, 105, 209

Jordán, A., Côté, P., Blakeslee, J. P., et al. 2005, ApJ, 634, 1002

Kinney, A. L., Calzetti, D., Bohlin, R. C., et al. 1996, ApJ, 467, 38

Kodama, T., \& Arimoto, N. 1997, A\&A, 320, 41

Kravtsov, A. V., Gnedin, O. Y., \& Klypin, A. A. 2004, ApJ, 609, 482

Kuzio de Naray, R., McCaugh, S. S., \& de Blok, W. J. G. 2004, MNRAS, 355, 887

Lobo, C., Biviano, A., Durret, F., et al. 1997, A\&A, 317, 385

López-Cruz, O., Yee, H. K. C., Brown, J. P., Jones, C., \& Forman, W. 1997, ApJ, 475, 97

López-Cruz, O., Barkhouse, W. A., \& Yee, H. K. C. 2004, ApJ, 614, 679 
Mateo, M. 1998, ARA\&A, 36, 435

McGaugh, S. S., Rubin, V. C., \& de Blok, W. J. G. 2001, AJ, 122, 2381

McCracken, H. J., Radovich, M., Bertin, E., et al. 2003, A\&A, 409, 835

Moore, B., Katz, N., Lake, G., Dressler, A., \& Oemler, A. Jr. 1996, Nature, 379

$$
613
$$

Moore, B., Lake, G., \& Katz, N. 1998, ApJ, 495, 139

Neumann, D. M., Lumb, D. H., Pratt, G. W., \& Briel, U. G. 2003, A\&A, 400, 811

Odell, A., Schombert, J., \& Rakos, K. 2002, AJ, 124, 3061

O’Neil, K., Bothun, G. D., \& Cornell, M. E. 1997, AJ, 113, 1212

Roberts, S., Davies, J., Sabatini, S., et al. 2004, MNRAS, 352, 478

Sabatini, S., Davies, J., van Driel, W., et al. 2005, MNRAS, 357, 819

Sandage, A. 1972, ApJ, 176, 21

Schombert, J. M. 1992, Proc. 5th International Workshop of Oss. Capodimonte, September 3-7, 1990, Italy (Kluwer Academic Publishers), ed. Longo et al., ASS Library, 178, 53
Schombert, J. M., Bothun, G. D., Schneider, S. E., \& McGaugh, S. S. 1992, AJ, 103, 1107

Secker, J., Harris, W. E., \& Plummer, J. D. 1997, PASP, 109, 1377

Sprayberry, D., Impey, C. D., \& Irwin, M. J. 1996, ApJ, 463, 535

Terlevich, A. I., Caldwell, N., \& Bower, R. G. 2001, MNRAS, 326, 1547

Thompson, L. A., \& Gregory, S. A. 1993, AJ, 106, 2197

Trentham, N. 1998, MNRAS, 293, 71

Ulmer, M. P., Bernstein, G. M., Martin, D. R., et al. 1996, AJ, 112, 2517

van den Bergh, S., Morbey, C., \& Pazder, J. 1991, ApJ, 375, 594

Vazdekis, A., Kuntschner, H., Davies, R. L., et al. 2001, ApJ, 551, L127

White, S. D. M., \& Rees, M. J. 1978, MNRAS, 183, 341

White, S. D. M., \& Frenk, C. S. 1991, ApJ, 379, 52

Zaritsky, D., Kennicutt, R. C., \& Huchra, J. P. 1994, ApJ, 420, 87 